Retraction

\title{
Retraction: Hsia et al. Post-Intake of $S$-Ethyl Cysteine and $S$-Methyl Cysteine Improved LPS-Induced Acute Lung Injury in Mice. Nutrients 2016, 8, 507
}

\author{
Te-chun Hsia ${ }^{1,2}$, Mei-chin Yin ${ }^{3,4, *}$ and Nutrients Editorial Office ${ }^{5}$ \\ 1 Department of Respiratory Therapy, China Medical University, Taichung City 40402, Taiwan; \\ derrick.hsia@msa.hinet.net \\ 2 Department of Internal Medicine, China Medical University Hospital, Taichung City 40402, Taiwan \\ 3 Department of Nutrition, China Medical University, 91, Hsueh-shih Rd., Taichung City 40402, Taiwan \\ Department of Health and Nutrition Biotechnology, Asia University, Taichung City 41354, Taiwan \\ 5 MDPI AG, St. Alban-Anlage 66, 4052 Basel, Switzerland; nutrients@mdpi.com \\ * Correspondence: mcyin@mail.cmu.edu.tw; Tel.: +886-4-2205-3366 (ext. 7510); Fax: +886-4-2206-2891
}

Received: 17 May 2017; Published: 23 May 2017

The integrity of several Western blot bands in Figures 3 and 4 [1] has been called into question. As a result, the authors of this article have decided to retract it and will repeat the entire analysis, to be submitted as a new article. We apologize to readers of Nutrients for any inconvenience caused.

Nutrients is a member of the committee on publication ethics (COPE) and takes the integrity of publications very seriously. In the interests of correcting the research literature, [1] will be marked as retracted.

\section{References}

1. Hsia, T.-C.; Yin, M.-C. Post-intake of S-ethyl cysteine and S-methyl cysteine improved LPS-induced acute lung injury in mice. Nutrients 2016, 8, 507. [CrossRef] [PubMed] 